\title{
REUSING FORMAL MODELS
}

\section{Domain capitalization via formalization}

\author{
Denis Sabatier \\ ClearSy, France \\ E-Mail: denis.sabatier@clearsy.com
}

\section{Introduction}

Formal models of all kinds are more and more widely used in a growing number of industrial projects. In such projects, people are looking for any means to master complexity, and semi-formal or formal models seem to be the only technical option, after management and organization improvements have been pushed to their limits.

But writing useful formal models is not an easy task. So there comes the natural idea of making this task more feasible by reusing previous work; with differentoptions:

- Building libraries of predefined models;

- Defining domain specialized formal languages;

- Defining domain specialized method or design patterns.

Which option should work best, and under what conditions? This is what I will try to guess here.

\section{Two kinds of model usage}

Let's examine more closely how formal models can be used in projects. There are many ways, but I think all of them fall in one of the two following categories: Detailed Component Specifications or High Level Requirements.

\subsection{Detailed Component Specifications}

This is what we do when use formal models to describe in an (hopefully!) non-ambiguous way all the computations done in a software module, for example. We can use sets, relationships, state diagrams, events order laws to describe the behavior of a component in any situation. 
For example, in a railway system with a track divided in blocks, we can describe how each block is labeled as occupied or free by the control system. Usually, such a system will use different kinds of truck detectors on the length of the block and at its boundaries with adjacent blocks. A block gets occupied if one of these detectors detects something. It gets free only when the detector at one of the boundaries changes from "detection" to "no detection", provided that the next block's boundary detector detects something, and that all other detectors for the block say "no detection", and that no alarm conditions have occurred, etc. All these can be described using either state diagrams, booleans equations, sets and relationships.

Although such detailed models might look like programs, they are still far simpler than actual code, especially if the formal representation uses abstract enough constructs. So here is the first, and maybe the main benefit: it's possible to write such detailed formalized specifications from the global requirements with a limited risk, where writing actual code directly from these requirements would lead to catastrophic errors. Even if the formal model contains all the data to actually do the module's task, not having to be runnable gives the ability to get rid of implementation details.

The next benefit is that it should be possible to derive the actual system from such formalized detailed specification. We can use a code generator, or use a way to obtain the code in a proven way from the detailed specifications.

Successful applications of such detailed formalized specifications are numerous. A very remarkable one that I know quite well is STS's use of the B Method (STS: Siemens Transportation Systems), with in particular the METEOR automated train development. In Meteor, the code was obtained by proven successive refinements from a detailed formalized software specification, producing a system that is still now considered as completely bug free since the first version, although unit tests were bypassed. STS improved further the process by automating the lowest refinement levels: a tool named EDITHB is now used to automatically build these levels from configurable design patterns (currently used in STS's New York projects).

Components detailed formalized specification can be used even for non software parts: ClearSy uses it to describe the functional principles of PEUGEOT cars, as an input for the development of the repair tool knowledge databases.

Of course, with this kind of formal models, it is still possible that the formally described behavior might not be what the user really needs. In our previous example with free / occupied blocks, will the proposed algorithm leads to a "good", safe and available train system? Having a formalized expression of these algorithms certainly helps, but this is not a proof that the system works as it should in the most general sense.

So this "detailed component specification" use of formal methods works well when the specifications are well defined and stabilized. In projects where 
what each component should do is still unclear, premature detailed formalizations can be harmful. I have a personal experience for this: once I had to develop a small tool for Peugeot. Lacking the time to correctly examine the users' needs, I wrote directly a detailed specification in B language. From this specification, I was able to develop in a few days a tool that seemed bug free, but we soon discovered that its results were useless, although there were what was requested in my specification! Both the development and the formal specification work time were lost. Such an example might not transpose to large industrial projects, but most of them still suffer from lack of stabilized specifications.

\subsection{Higher Level Specification}

If we are unsure of what functionalities are really needed, detailed formal specification might be premature. We can then use formal models to point out what is really needed. In our previous example (free / occupied blocks), what are the true requirements? One could say:

"block occupied" should be equivalent to the physical fact that there is some material on the block part of the track.

Such a requirement is clearly not what is done by an algorithm such as the one presented above. Firstly, it is not always possible to detect material on the track because sensors might be faulty, so the above requirement is infeasible. A better requirement would be that if all sensors are OK, block occupied represents material on the track, but if one or more sensors fail, the block is declared as occupied. Even then, alarm conditions that can stop the block release and necessary overlapping zones at block boundaries certainly exists, so this requirement shows up as a quite naive one. More, a fugitive fault of a sensor might cause a useless and dangerous emergency brake with such an "occupied if failure" naive requirement.

In fact, the block occupation and release algorithm has been carefully chosen so that the system will preserve safety with a certain amount of faulty sensors, that it will tolerate trucks with certain dimensional characteristics, that it will preserve minimum safety if a truck derails by not releasing the block... We see that finding the true requirement forces an "expansion" of the scope by always asking questions like:

- Why do we want this property?

- What can we assume on the external environment?

By recursively using these two questions, we would rapidly end up in "world level" requirements, like what is requested from any transportation system and whether we should build a train or a plane. So it is necessary to decide the limits of the scope, for example: 
- Assuming that we have a control system that is certainly safe if a free block is really free of material with a certain safety gap;

- Assuming that the sensors will comply to a certain functional model and a certain failure behavior;

- Assuming that no material will never appear in the middle of the track, or be in a shape such that sensors might not detect it;

- $\ldots$

- Then the block control algorithm should be such that ...(and so on).

Trying to state formally the true requirements of a badly specified component requires the ability to expand the scope both in abstraction level and in environment knowledge, and the skill to stop this expansion at the right volume. This is not a task for beginners or newcomers in the field. Only experienced people with the proper authority on what should be assumed and what should be changed will have the best chances of success.

Generally, there is a tendency to keep the abstraction level too low and to assume too much on the environment and its stability. Things that seem so certain today become false sooner than expected... The small tool for Peugeot that I cited above as an example of too early detailed formalization was improved to satisfaction, then thrown away because a full automation was finally decided for the data generation that this tool was designed to help.

Thus, higher level models should be done with a carefully chosen larger scope. Another problem with this kind of models is that they require abstract variables: variables meant to represent physical facts but not directly measurable. For instance, the notion of detectable material in our free / occupied block example. Such variables require very precise definition, otherwise models become links between vague notions that are no longer unambiguously related to reality.

One more problem with higher level models is the omnipresence of quantitative criteria. For instance, in our block example declaring a block as occupied because of failures should be restricted to the minimum. Formalizing quantitative requirements like this one is useless, as in most cases we cannot prove a design as being the optimal one.

High skills, large scopes, abstract notions and unprovable quantitative requirements are claims against higher level formal models. Why not keep all this informal? Despite the difficulties, I still believe that higher level models are worth it, because if not we are restricted to finding proper design by trial and error and careful examination of requirements doomed to stay informal; this would be giving up the intellectual mastering of complex problems. To succeed with higher level models, let's not make them too high and let's not require them to cover all aspects. 
Industrial use of higher level models is not yet common. Case studies on examples from industry show that designing a system with important high level properties formalized and proven is possible and affordable; and we use higher level "modelization" at ClearSy in real projects as internal initiatives. We believe that in the near future, we will see larger scale applications for this kind of formalization.

\section{Clues for capitalization}

After this examination of formal model types, let's go back to how to reuse and capitalize on formal models.

\section{1 $\quad$ Reusing detailed models}

If we consider formal models like the one that describes the algorithm used for block occupation and release, it is quite clear that such models are valuable only for what they are made for. Of course, careful study of these models will help building new ones for a different system, but as they constitute the company's know-how, it is unlikely that exchanges will go beyond the development team where models were originally written, maybe even not beyond the very person who wrote each model. Reuse always works well within a team of one person...

As a side effect of being developed from a detailed formalized specification, the obtained modules have a well known behavior. So instead of trying to reuse formal specifications as specifications, a better way would be to reuse the developed modules as COTS, claiming that their behavior is well known. Equipment manufacturers could thus sell components from their own systems as formally defined reusable parts. They could hope that by common use in other systems, their components and their architecture might become a standard. This could counter-balance the necessary diffusion of valuable knowhow. In this idea of making components reusable thanks to formal specification, it's probably better to extract components out of real operational projects only. Specifying reusable components without the help of a real need is a very difficult challenge.

Another way of capitalization would be designing domain specific formal languages. As each domain has its specialized technical terms, each domain could have its specialized formal language. The problem is that in the same domain specialized formal languages could rapidly become different between companies, the same way technical terms often differ, and this could lead to harmful obscurantism. I feel that formal languages should remain open, at least as a necessary condition for the diffusion of formally specified reusable components. 


\subsection{Reusing higher level models}

Are higher level models reusable? In the occupied / free block example, the higher model would be the one that says all the assumptions about sensors type and performances, rolling stock characteristics, etc, to define what the necessary occupation property should be. In other words, such a model would be a pre-digested understanding of what should be considered to design a safe and available train localisation system, with the choice of any type of rolling stock and sensors (within the assumptions limits) that the up-to-date technology can offer. This is naturally useful for any case that falls within the limits of the assumptions made on the environment. Naturally, the more abstract the models are, the more reusable they are.

Probably, the formal design between highest level models to detailed specifications might not be reusable, due to know-how confidentiality problems or simply because the next user will design differently. So the most reusable part is at the top, applicable to all analog cases in the domain. We obtain domain specific models which are pre-digested understanding of good, out of the field domain notions. This is reusable by construction.

The main difficulty for reusing higher level models is obviously that there are only few actually written. In addition to the technical difficulties depicted before, higher level models cause organisation problems: they require the collaboration of domain experts and "modelization" experts. Domain experts have a long time established view of their field, they won't easily accept any change in the way their notions are expressed: this would interfere with their very mental constructs. "Modelization" experts tend to reinvent terms and notions differently, which is a bad idea when unnecessary. To avoid these pitfalls, a common solution is trying to build a tool that would allow domain experts to become "modelization" experts as well. I'm afraid that this solution will always limit the quality of models.

If all these difficulties are overcome, let's imagine an equipment manufacturer who designs products with a first phase of higher level "modelization" gathering domain experts and formalization experts. How and why would this manufacturer propose his top level models for reuse by others? The immaterial nature of these models makes it difficult to view them as sell-able items. The only way I can think of is that certification organisms could collect these models as certification templates, a bit like the Common Criteria in the domain of IT system security. Quite often, the certification organism is the company that will use the final product; such companies gather the best possible set of domain experts. So if top level models could be created in phases where the manufacturer and the final exploitation company collaborate, these models would be certification templates from the start. The final exploitation company 
would naturally provide them to increase the competition between manufacturers and get better prices.

\section{Who will pay for that?}

Both for detailed or higher level models, there are clues for re-usability. But who will pay for that anyway? Formal models in projects are a bit like fluor in toothpaste: it costs more at the beginning and one can only see the effects after a long time.

Even if the perspective of formally specified reusable components and formally defined common certification models is nice, the first step to this is to collect data about the development and exploitation of complex systems and relate them to how much formalization was used. Only a big amount of such studies by independent organisms can prove the return over investment of formal methods. In the meantime, companies who regard the existing evidence as sufficient can use formal methods internally for the benefit of their customers, this is what we do at ClearSy. These companies can start the reuse process, probably more with reusable components than with higher level models.

\section{Conclusion}

There are two ways of reusing formal models that seem best promising:

- Providing components out of projects with detailed level formal specifications, claiming that the formal specifications make them reusable;

- Providing higher level formal models as certification criteria.

The development of these reuse possibilities should follow the spreading of formal methods. Let's see them as additional reasons to promote the use of formal methods. 